pam, Temazepam oder Triazolam geeignet, nicht jedoch lang wirksame Vertreter wie Bromazepam, Diazepam oder Lormetazepam - diese könnten die Schmerzen mitunter verstärken. Chloralhydrat und Antihistaminika hält Geisler bei Schmerzpatienten ebenfalls für wenig geeignet.
Gerade bei Patienten mit neuropathischen Schmerzen (Abb. 1) ist Pregabalin eine interessante Option. Das Schmerzmittel hat bereits einen gut schlaffördernden Effekt, hier müsse möglicherweise nur die Hauptdosis am Abend verabreicht werden, um von dieser Wirkung zu profitieren. Generell sollten Ärzte mit
Pregabalin aufgrund des Abhängigkeitspotenzials aber vorsichtig umgehen. Die Entzugserscheinungen seien oft schlimmer als die bei einer Benzodiazepinabhängigkeit, sagte der Schlafmediziner.

Thomas Müller

Symposium 4: Schlaf und Schmerz. 23. Jahrestagung der DGSM, Mainz, 3.-5.12.2015

\title{
Der Kopf scheint im Schlaf zu explodieren
}

\author{
Einige Patienten haben vor allem nachts Kopfschmerzen. Oft stecken Atem- \\ probleme dahinter, mitunter aber auch recht exotische Phänomene. Dann \\ reicht manchmal eine Tasse Kaffee am Abend, um die Schmerzen fernzuhalten.
}

Se chlafstörungen bei Kopfschmerzpatienten sind keine Seltenheit. Nach epidemiologischen Untersuchungen finden drei von vier Kopfschmerzpatienten keinen erholsamen Schlaf, erläuterte Professor Svenja Happe von der Klinik für Neurologie in Telgte. Die Gründe dafür sind vielfältig: Kopfschmerzmedikamente können den Schlaf stören, auch scheinen Patienten mit Kopfschmerzen eine Neigung für bestimmte Schlafstörungen zu haben, sagte die Neurologin auf der Tagung der Deutschen Gesellschaft für Schlafforschung und Schlafmedizin (DGSM) in Mainz. Als Beispiel nannte sie eine erhöhte Prävalenz von Parasomnien bei jungen Migränepatienten. Auch gebe es Hinweise auf einen Zusammenhang zwischen Narkolepsie und Migräne. In eigenen Untersuchungen konnte Happe eine erhöhte Prävalenz des Restless-legs-Syndroms bei Migränepatienten feststellen: Von 411 Patienten waren $17 \%$ betroffen, in einer Kontrollgruppe ohne Migräne trat ein RLS nur bei $6 \%$ der Teilnehmer auf. Bei Patienten mit Spannungs- und Clusterkopfschmerz fanden die Forscher um Happe hingegen keine erhöhte RLS-Prävalenz.

\section{Attacken beginnen meist im REM-Schlaf}

Ein besonderes Problem können auch Kopfschmerzen darstellen, die im Schlaf auftreten. Dies ist sowohl bei Migräneals auch bei Clusterkopfschmerzen nicht selten der Fall. Clusterkopfschmerzen treten $\mathrm{zu}$ rund $60 \%$ im Schlaf auf, bei rund jedem zehnten Patienten sogar aus- schließlich im Schlaf, sagte Happe. Die Attacken beginnen meist im REM-Schlaf und im vierten Schlafstadium. Bei nächtlichen Clusterkopfschmerzen hätten die Patienten in den meisten Fällen auch eine schlafbezogene Atemstörung - allerdings nur bei episodischem Clusterkopfschmerz. Meist (bei 65-85\%) treten die Attacken nach einer Sauerstoffentsättigung und bei erhöhtem $\mathrm{CO}_{2}$ Partialdruck auf. Häufig ließen sich im aktiven Cluster auch Apnoen beobachten. Umgekehrt leidet ein Drittel bis die Hälfte der Patienten mit einem obstruktiven Schlafapnoesyndrom auch unter Kopfschmerzen, wobei die Schmerzen mit der Ausprägung der Schlafapnoe zunehmen. Eine naheliegende Therapie ist dann die Überdruckbeatmung. Es gebe inzwischen einige Hinweise, wonach die CPAP auch gegen Clusterkopfschmerz bei Schlafapnoe wirksam ist. Happe gab jedoch zu bedenken, dass die meisten der Apnoen beim Clusterkopfschmerz zentral und nicht obstruktiv bedingt sind.

Eine Besonderheit sind Kopfschmerzen, die ausschließlich im Schlaf auftreten. Die Neurologin nannte hier vor allem den primären schlafgebundenen Kopfschmerz, der die Betroffenen aus dem Schlaf reißt. Nach dem Aufwachen hält der Schmerz in der Regel mindestens eine Viertelstunde und maximal vier Stunden an. Die Diagnosekriterien sind erfüllt, wenn über drei Monate hinweg mindestens zehn nächtliche Attacken pro Monat auftreten und sich keine kranialen oder autonomen Ursachen fin- den lassen. Betroffen sind meist ältere Menschen, zu zwei Dritteln Frauen, bei rund $60 \%$ treten die Schmerzen bilateral auf. Die Attacken beginnen in der Regel ebenfalls im REM-Schlaf. Vermutet werden Veränderungen im suprachiasmatischen Kerngebiet.

Erstaunlicherweise scheint Koffein bei einigen Patienten gut prophylaktisch zu wirken: Mit einer Tasse Kaffee am Abend blieben viele die Nacht über schmerzfrei. Auch für NSAR, Lithium (300-600 mg), Indometacin $(50-200 \mathrm{mg})$ sowie Flunarizin $(5-10 \mathrm{mg})$ gebe es Berichte zur Wirksamkeit.

\section{Schrecklicher Knall im Kopf}

Ebenfalls schlafgebunden ist der „turtle headache“. Diese Kopfschmerzform wird vor allem morgens nach dem Wiedereinschlafen beobachtet. Häufig haben sich die Betroffenen dabei ein Kissen über den Kopf gezogen, liegen unter der Bettdecke oder haben einen Arm auf dem Gesicht. Dies deute auf eine Hypoxie oder Hyperkapnie als Mechanismus.

Gelegentlich suchen auch Patienten mit "exploding head syndrom“ einen Neurologen auf: Die Patienten hören einen lauten Knall und haben das Gefühl, etwas in ihrem Kopf ist explodiert. Meist tritt das Phänomen beim Schlaf-WachÜbergang oder beim Erwachen in der Nacht auf. Die Patienten haben in der Regel jedoch keine Schmerzen, aber ein ausgeprägtes Furchtgefühl. Gelegentlich können Lichtblitze und Myoklonien das Ereignis begleiten. Hier komme es vor allem darauf an, die Patienten zu beruhigen. Das Syndrom scheine nicht auf einer bedrohlichen Ursache zu beruhen und sei möglicherweise mit dem Pavor nocturnus verwandt, erläuterte Happe.

Thomas Müller

Symposium 4: Schlaf und Schmerz. 23. Jahrestagung der DGSM, Mainz, 3.-5.12.2015 